Applied Mathematical Sciences, Vol. 9, 2015, no. 90, 4469 - 4477

HIKARI Ltd, www.m-hikari.com

http://dx.doi.org/10.12988/ams.2015.53279

\title{
Spherical Indicatrix Curves of Spatial Quaternionic Curves
}

\author{
Süleyman Şenyurt ${ }^{1}$ \\ Faculty of Arts and Sciences, Department of Mathematics \\ Ordu University, 52100, Ordu, Turkey \\ Luca Grilli \\ Department of Economics \\ University of Foggia, Foggia, Italy \\ Copyright (c) 2015 Süleyman Şenyurt and Luca Grilli. This article is distributed under \\ the Creative Commons Attribution License, which permits unrestricted use, distribution, \\ and reproduction in any medium, provided the original work is properly cited.
}

\begin{abstract}
In this paper, the spherical indicatrix curves drawn by quaternionic frenet vectors are computed. Also the quaternionic geodesic curvatures of the spherical indicatrix curves to $E^{3}$ and $S^{2}$ are found.
\end{abstract}

Mathematics Subject Classification: 11R52, 53A04

Keywords: Real quaternion, Spatial quaternion, Indicatrix curve

\section{Introduction}

Quaternions were discovered, for the first time in 1843, by the Irish mathematician Sir William R. Hamilton [2]. Hamilton wanted to generalize complex numbers in order to be used in geometric optics. In 1987, Bharathi and Nagaraj defined the quaternionic curves in $\mathbb{E}^{3}$ and $\mathbb{E}^{4}$, they studied the differential geometry of space curves and introduced Frenet frames and formulae by using quaternions [4]. About a decade later, quaternionic inclined curves have been

\footnotetext{
${ }^{1}$ Corresponding author
} 
defined and harmonic curvatures studied by Karadăg and Sivridağ [6]. Tuna and Çöken have studied quaternion valued functions and quaternionic inclined curves in the semi-Euclidean space $\mathbb{E}_{2}^{4}$ [1]. They have given the Serret-Frenet formulae and they have defined quaternionic inclined curves and harmonic curvatures for the quaternionic curves in the semi-Euclidean space. Quaternionic rectifying curves have been studied by Güngör and Tosun, [5]. Şenyurt and Çalışkan have founded the Darboux vector of the spatial quaternionic curve according to the Frenet frame. Then, they calculated the curvature and torsion of the spatial quaternionic Smarandache curve formed by the unit Darboux vector with the normal vector, [7].

\section{Preliminaries}

In this section, we give the basic elements of the theory of quaternions and quaternionic curves. A more complete elementary treatment of quaternions and quaternionic curves can be found in [3] and [4], respectively. A real quaternion $q$ is an expression of the form

$$
q=d+a e_{1}+b e_{2}+c e_{3}
$$

where $a, b, c \in \mathbb{R}$ and $e_{i}, 1 \leq i \leq 3$, are quaternionic units which satisfy the non-commutative multiplication rules

$$
\left\{\begin{array}{l}
e_{1}^{2}=e_{2}^{2}=e_{3}^{2}=e_{1} \times e_{2} \times e_{3}=-1, \quad e_{1}, e_{2}, e_{3} \in \mathbb{R}^{3} \\
e_{1} \times e_{2}=e_{3}, e_{2} \times e_{3}=e_{1}, e_{2} \times e_{3}=e_{1}
\end{array}\right.
$$

The algebra of the quaternions is denoted by $Q$ and its natural basis is given by $\left\{e_{1}, e_{2}, e_{3}\right\}$. A general quaternion can be given by the form

$$
q=S_{q}+V_{q}
$$

where $S_{q}=d$ is the scalar part and $V_{q}=a e_{1}+b e_{2}+c e_{3}$ is the vector part of $q$. The Hamilton conjugate of $q=S_{q}+V_{q}$ is defined by $\bar{q}=S_{q}-V_{q}$. Summation of two quaternions $q_{1}=S_{q_{1}}+V_{q_{1}}$ and $q_{2}=S_{q_{2}}+V_{q_{2}}$ is defined as $q_{1} \oplus q_{2}=\left(S_{q_{1}}+S_{q_{2}}\right)+\left(V_{q_{1}}+V_{q_{2}}\right)$. Multiplication of a quaternion $q=S_{q}+V_{q}$ with a scalar $\lambda \in \mathbb{R}$ is identified as $\lambda \odot q=\lambda S_{q}+\lambda V_{q}$. If $\|q\|=1$, then $q$ is called unit quaternion. Let $q_{1}=S_{q_{1}}+V_{q_{1}}=d_{1}+a_{1} e_{1}+b_{1} e_{2}+c_{1} e_{3}$ and $q_{2}=S_{q_{2}}+V_{q_{2}}=d_{2}+a_{2} e_{1}+b_{2} e_{2}+c_{2} e_{3}$ be two quaternions in, then the quaternion product of $q_{1}$ and $q_{2}$ is given by

$$
\begin{aligned}
q_{1} \times q_{2} & =d_{1} d_{2}-\left(a_{1} a_{2}+b_{1} b_{2}+c_{1} c_{2}\right)+\left(d_{1} a_{2}+a_{1} d_{2}+b_{1} c_{2}-c_{1} b_{2}\right) e_{1} \\
& +\left(d_{1} b_{2}+b_{1} d_{2}+b_{1} a_{2}-a_{1} b_{2}\right) e_{2}++\left(d_{1} c_{2}+c_{1} d_{2}+a_{1} b_{2}-b_{1} a_{2}\right) e_{3}
\end{aligned}
$$


or

$$
q_{1} \times q_{2}=S_{q_{1}} S_{q_{2}}-\left\langle V_{q_{1}}, V_{q_{2}}\right\rangle+S_{q_{1}} V_{q_{2}}+S_{q_{2}} V_{q_{1}}+V_{q_{1}} \wedge V_{q_{1}}
$$

where $\langle$,$\rangle and \wedge$ denote the inner product and vector product in Euclidean 3 -space.

In this space it is defined a symmetric real-valued, non-degenerate, bilinear form as follows:

$$
\langle,\rangle_{\left.\right|_{Q}}: Q \times Q \rightarrow \mathbb{R},\left.\left\langle q_{1}, q_{2}\right\rangle\right|_{Q}=\frac{1}{2}\left(q_{1} \times \overline{q_{2}}+q_{2} \times \overline{q_{1}}\right)
$$

which is called the quaternion inner product. As a result the norm of $q$ is

$$
N(q)=\sqrt{q \times \bar{q}}=\sqrt{a^{2}+b^{2}+c^{2}+d^{2}} .
$$

The element $q$ is called a spatial quaternion whenever $q+\bar{q}=0$ and called a temporal quaternion whenever $q-\bar{q}=0$. A general quaternion $q$ can be given as $q=\frac{1}{2}(q+\bar{q})+\frac{1}{2}(q-\bar{q})$. The three-dimensional Euclidean space is identified with the space of spatial quaternions [4].

The space $Q_{H}=\{q \in Q \mid q+\bar{q}=0\}$ is defined in an obvious manner. Let $I=[0,1]$ be an interval in the real line $\mathbb{R}$ and $s \in I$ be the arc-length parameter along the smooth curve

$$
\gamma:[0,1] \rightarrow Q_{H}, \quad \gamma(s)=\sum_{i=1}^{3} \gamma_{i}(s) e_{i}
$$

The tangent vector $\gamma^{\prime}(s)=t(s)$ has unit length $\|t(s)\|=1$ for all $s$.

Let $\gamma:[0,1] \rightarrow Q_{H}$ be a differentiable spatial quaternions curve with arclength parameter $s$ and $\left\{t(s), n_{1}(s), n_{2}(s)\right\}$ be the Frenet frame of $\gamma$ at the point $\gamma(s)$, where

$$
\left\{\begin{array}{l}
t(s)=\gamma^{\prime}(s) \\
n_{1}(s)=\frac{\gamma^{\prime \prime}(s)}{N\left(\gamma^{\prime \prime}(s)\right)} \\
n_{2}(s)=t(s) \times n_{1}(s),
\end{array}\right.
$$

and if the curve $\gamma(s)$ is a non unit speed curve then we say that

$$
\left\{\begin{array}{l}
t(s)=\frac{\gamma^{\prime}(s)}{\nu(s)}, \nu(s)=N\left(\gamma^{\prime}(s)\right) \\
n_{1}(s)=n_{2}(s) \times t(s) \\
n_{2}(s)=\frac{\gamma^{\prime}(s) \times \gamma^{\prime \prime}(s)+\nu(s) \nu^{\prime}(s)}{N\left(\gamma^{\prime}(s) \times \gamma^{\prime \prime}(s)+\nu(s) \nu^{\prime}(s)\right)} .
\end{array}\right.
$$


Let $\left\{t(s), n_{1}(s), n_{2}(s)\right\}$ be the Frenet frame of $\gamma(s)$. Then Frenet formula, curvature and the torsion are given by

$$
\left\{\begin{array}{l}
t^{\prime}(s)=k(s) n_{1}(s) \\
n_{1}{ }^{\prime}(s)=-k(s) t(s)+r(s) n_{2}(s) \\
n_{2}{ }^{\prime}(s)=-r(s) n_{1}(s)
\end{array}\right.
$$

and

$$
\left\{\begin{array}{l}
k(s)=\frac{N\left(\gamma^{\prime}(s) \times \gamma^{\prime \prime}(s)+\nu(s) \nu^{\prime}(s)\right)}{\nu(s)^{3}} \\
r(s)=\frac{\left\langle\gamma^{\prime}(s) \times \gamma^{\prime \prime}(s), \gamma^{\prime \prime \prime}(s)\right\rangle_{\left.\right|_{Q}}}{\left[N\left(\gamma^{\prime}(s) \times \gamma^{\prime \prime}(s)+\nu(s) \nu^{\prime}(s)\right)\right]^{2}},
\end{array}\right.
$$

where $t(s), n_{1}(s), n_{2}(s)$ are, respectively, the unit tangent, the unit principal normal and the unit binormal vector of a quaternionic curve [4]. The functions $k, r$ are called the principal curvature and the torsion, respectively.

Considering the spatial quaternions curve $\gamma:[0,1] \rightarrow Q_{H}$, the moving frame $\left\{t(s), n_{1}(s), n_{2}(s)\right\}$ moves with a certain angular velocity around each axis for any $s \in[0,1]$. This axis is called instantaneous rotation axis of the spatial quaternionic curve. The Darboux axis vector in the direction indicated by $\mathrm{D}$ in defined as follows:

$$
D=r t+k n_{2} .
$$

Let $D$ be the instantaneos Pfaff vector of curve $\gamma$. Let denote the angle between $D$ and $n_{2}$ with $\varphi$,

$$
\cos \varphi=\frac{k}{\sqrt{k^{2}+r^{2}}}, \quad \sin \varphi=\frac{r}{\sqrt{k^{2}+r^{2}}}
$$

The unit vector of quaternionic Darboux vector [7] is indicated by $w$ :

$$
w=\frac{D}{N(D)}=\sin \varphi t+\cos \varphi n_{2} .
$$

\section{Spherical Indicatrix Curves of Spatial Quater- nionic Curves}

In this section we find the arc lenght of $(t),\left(n_{1}\right),\left(n_{2}\right)$ and $(w)$ for the Spherical Indicatrix Curves, we compute the geodesic curvatures in $E^{3}$ and $S^{2}$.

We indicate the arc length for $(t)$ with $s_{t}$, that is 


$$
\begin{aligned}
s_{t} & =\int_{0}^{s} N\left(\frac{d t}{d s}\right) d s \\
& =\int_{0}^{s} N\left(k n_{1}\right) d s \\
& =\int_{0}^{s} \sqrt{\frac{1}{2}\left(k n_{1} \times \overline{k n_{1}}\right)+\left(k n_{1} \times \overline{k n_{1}}\right)} d s \\
& =\int_{0}^{s} k d s .
\end{aligned}
$$

If the arc length for $\left(n_{1}\right),\left(n_{2}\right)$ and $(w)$ are $s_{n_{1}}, s_{n_{2}}$ and $s_{w}$, we have:

$$
\begin{aligned}
s_{n_{1}} & =\int_{0}^{s} N\left(\frac{d n_{1}}{d s}\right) d s \\
& =\int_{0}^{s} N\left(-k t+r n_{2}\right) d s \\
& =\frac{1}{\sqrt{2}} \int_{0}^{s} \sqrt{\left(\left(-k t+r n_{2}\right) \times\left(\overline{-k t+r n_{2}}\right)+\left(-k t+r n_{2}\right) \times\left(\overline{-k t+r n_{2}}\right)\right)} d s \\
& =\frac{1}{\sqrt{2}} \int_{0}^{s} \sqrt{2\left(k^{2}+r^{2}\right)} d s \\
& =\int_{0}^{s} N(w(s)) d s,
\end{aligned}
$$




$$
\begin{aligned}
s_{n_{2}} & =\int_{0}^{s} N\left(\frac{d n_{2}}{d s}\right) d s \\
& =\int_{0}^{s} N\left(-r n_{2}\right) d s \\
& =\frac{1}{\sqrt{2}} \int_{0}^{s} \sqrt{\left(-r n_{2} \times \overline{-r n_{2}}\right)-\left(r n_{2} \times \overline{-r n_{2}}\right)} d s \\
& =\int_{0}^{s} r d s, \\
s_{w} & =\int_{0}^{s} N\left(\frac{d w}{d s}\right) d s \\
& =\int_{0}^{s} N\left(\frac{d\left(\sin \varphi t+\cos \varphi n_{2}\right)}{d s}\right) d s \\
& =\int_{0}^{s} N\left(\varphi^{\prime}\left(\cos \varphi t-\sin \varphi n_{2}\right)\right) d s \\
& =\int_{0}^{s} \varphi^{\prime} d s .
\end{aligned}
$$

Let $\alpha_{t}\left(s_{t}\right)=t(s)$ be a unit speed regular spherical curves. We denote $s_{t}$ as the arc-lenght parameter of tangents indicatrix $(t)$

$$
\alpha_{t}\left(s_{t}\right)=t(s)
$$

Let us consider the tangent vector $t_{t}$ of curve $(t)$. Supposing that the tangent vector of the $(t)$ geodeseic curvature is $\Lambda_{t}$, we get

$$
\Lambda_{t}=N\left(D_{t_{t}} t_{t}\right) .
$$

Differentiating (3.5), we have

$$
\frac{d \alpha_{t}}{d s_{t}} \frac{d s_{t}}{d s}=t^{\prime}(s)
$$


and

$$
t_{t} \frac{d s_{t}}{d s}=k n_{1}
$$

From the equation (3.7)

$$
t_{t}\left(s_{t}\right)=n_{1}(s) .
$$

Computing the derivatives and after some algebra we get,

$$
D_{t_{t}} t_{t}=-t+\frac{r}{k} n_{2}
$$

By substituting (3.5), we obtain

$$
\begin{aligned}
\Lambda_{t} & =\sqrt{\frac{1}{2}\left(-t+\frac{r}{k} n_{2}\right) \times \overline{\left(-t+\frac{r}{k} n_{2}\right)}}, \\
\Lambda_{t} & =\sqrt{1+\left(\frac{r}{k}\right)^{2}}
\end{aligned}
$$

or from the equation (2.13),

$$
\Lambda_{t}=\sec \varphi
$$

Likewise, let us suppose that the tangent vector of the curve $\left(n_{1}\right)$ is $t_{n_{1}}$. Supposing that $\Lambda_{n_{1}}$ is the geodesic curvature of $\left(n_{1}\right)$ at $E^{3}$, we get

$$
\Lambda_{n_{1}}=\sqrt{1+\left(\frac{\varphi^{\prime}}{N(w)}\right)^{2}} .
$$

Let us define the tangent vector of the curve $\left(n_{2}\right)$ as $t_{n_{2}}$. Supposing that $\Lambda_{n_{2}}$ is the geodesic curvature of $\left(n_{2}\right)$ at $E^{3}$, we get

$$
\begin{aligned}
& \Lambda_{n_{2}}=\sqrt{1+\left(\frac{k}{r}\right)^{2}} \\
& \Lambda_{n_{2}}=\csc \varphi .
\end{aligned}
$$

Following a similar approach, we consider the tangent vector is $t_{w}$ of the curve $(w)$. Supposing that $\Lambda_{w}$ is the geodesic curvature of $(w)$ at $E^{3}$, we get

$$
\Lambda_{w}=\sqrt{1+\left(\frac{N(w)}{\varphi^{\prime}}\right)^{2}} .
$$

Let find the geodesic curvatures of $(t),\left(n_{1}\right),\left(n_{2}\right)$ and $(w)$ to $S^{2}$. Supposing that the geodesic curvature for $(t)$ is $\Gamma_{t}$, it is $\Gamma_{t}=N\left(\bar{D}_{t_{t}} t_{t}\right)$. Because of the Gauss equation and $S\left(t_{t}\right)=t_{t}$, we have 


$$
\begin{aligned}
& \bar{D}_{t_{t}} t_{t}=D_{t_{t}} t_{t}+\left\langle S\left(t_{t}\right), t_{t}\right\rangle_{\left.\right|_{Q}} t \\
& \bar{D}_{t_{t}} t_{t}=D_{t_{t}} t_{t}+t, \\
& \bar{D}_{t_{t}} t_{t}=\frac{r}{k} n_{2}=\tan \phi n_{2} .
\end{aligned}
$$

Then,

$$
\Gamma_{t}=\tan \phi
$$

will be achieved. Likewise, Supposing that the geodesic curvature for $\left(n_{1}\right)$ is $\Gamma_{n_{1}}$, it is $\Gamma_{n_{1}}=N\left(\bar{D}_{t_{n_{1}}} t_{n_{1}}\right)$. Because $\left\langle S\left(t_{n_{1}}\right), t_{n_{1}}\right\rangle_{\left.\right|_{Q}}=1$ at the statement

$$
\begin{aligned}
& \bar{D}_{t_{n_{1}}} t_{n_{1}}=D_{t_{n_{1}}} t_{n_{1}}+\left\langle S\left(t_{n_{1}}\right), t_{n_{1}}\right\rangle_{\mid Q} t \\
& \bar{D}_{t_{n_{1}}} t_{n_{1}}=\frac{\varphi^{\prime}}{N(w)}\left(\sin \varphi t+\cos \varphi n_{2}\right)
\end{aligned}
$$

will be found out. Then,

$$
\Gamma_{n_{1}}=\frac{\varphi^{\prime}}{N(w)}
$$

will be achieved. Likewise, supposing that the geodesic curvatures for $\left(n_{2}\right)$ are $(w)$ are $\Gamma_{n_{2}}$ and $\Gamma_{w}$, these are $\Gamma_{n_{2}}=N\left(\bar{D}_{t_{n_{2}}} t_{n_{2}}\right)$ and $\Gamma_{w}=N\left(\bar{D}_{t_{w}} t_{w}\right)$. Then,

$$
\begin{aligned}
\Gamma_{n_{2}} & =\cot \varphi, \\
\Gamma_{w} & =\frac{N(w)}{\varphi^{\prime}} .
\end{aligned}
$$

will be achieved.

\section{References}

[1] A. Tuna, A. C. Çöken, On the quaternionic inclined curves in the semiEuclidean space, Applied Mathematics and Computation, 155(2), (2014), 373-389. http://dx.doi.org/10.1016/s0096-3003(03)00783-5

[2] Hamilton, W. R., Elements of Quaternions, I, II and III, Chelsea, New York, 1899.

[3] H. H. Hacisalioğlu, Hareket Geometrisi ve Kuaterniyonlar Teorisi, University of Gazi Press, 1983. 
[4] K. Bharath and M. Nagaraj, Quaternion Valued Function of a Real Variable Serret-Frenet Formulae, Indian J. Pure Appl. Math., 18(6), (1987), 507-511.

[5] M. A. Güngör and M. Tosun, Some characterizations of quaternionic rectifying curves, Differential Geometry - Dynamical Systems, Vol.13, Balkan Society of Geometers, Geometry Balkan Press, (2011), 89-100.

[6] M. Karadă̆, A.İ. Sivridă̆, Tek Değişkenli Kuaterniyon Değerli Fonksiyonlar ve Eğilim Çizgileri, Erc. Üniv. Fen Bil. Derg.,13, (1997), 23-36.

[7] S. Şenyurt and A. S. Çalışkan, An Application According to Spatial Quaternionic Smarandache Curve, Applied Mathematical Sciences, 9(5), (2015), 219-228. http://dx.doi.org/10.12988/ams.2015.411961

Received: April 8, 2015; Published: June 21, 2015 